\title{
PERFORMANCE ANALYSIS BETWEEN PCA AND ICA IN HUMAN FACE DETECTION
}

\author{
Md. Shahjahan Kabir ${ }^{1}$, Md. Solaiman Hossain ${ }^{2}$ and Dr. Rabiul Islam ${ }^{3}$ \\ ${ }^{1,2}$ Department of CSE, Dhaka Int. University (DIU), Dhaka, Bangladesh. \\ ${ }^{3}$ Associate Professor, Dept. of CSE, RUET, Rajshahi, Bangladesh.
}

\begin{abstract}
In the context of Face Recognition the paper compares between Principal Component Analyses (PCA) and Independent Component Analysis (ICA). In the psychological and algorithmic literature Principal Component Analyses (PCA) and Independent Component Analyses (ICA) are the basis of numerous studies. Classical technique in statistical data analyses is called Principal Component Analyses (PCA) and technique of array processing and data analysis is called Independent Component Analysis (ICA). This paper gives the better concept how each algorithms are worked. This concept helps to advance level experiment. Besides, those method is so strong others system and algorithm. In both case, ICA performs good but not as good as PCA. For Face Recognition Principal Component Analyses basis algorithm represents intelligent suction of a random search within a short time and its can detect to solve the problem.
\end{abstract}

\section{KEYWORDS}

Face Recognition, ICA, PCA, JAFFE database.

\section{INTRODUCTION}

Face Recognition is important part of computer vision. Besides the last some years Face Recognition is a powerful application. There are many commercial systems for human face detection is available now. For advance research efforts have been focused on video based face modelling or tracking, recognition and system integration. It's an important part of ability of human appreciation system. During the past years, Face Recognition has received increased evolution. Compression data is mandatory to use, Face Recognition better for this purpose. It has special technically views. Images can be compressed as structural features such as contours and regions. [1] It can be access through the human computer interface (HCI) in crowd surveillance. The earliest system on Face Recognition can be traced back at least to the 1950s in psychology and to the 1960s in the in the engineering literature. As perfect by the algorithms, this paper compares Principal Component Analysis (PCA) to Independent Component Analysis (ICA). Advantages of using Principal Component Analysis are data can be compressed image without harm any data. PCA can outperform over many other techniques when the size of database is small. In proposed algorithm the database is sub grouped using some features of interest in face. [2] The performances of appearance based statistical methods such as PCA and ICA are tested and compared for the recognition of coloured faces images in Face Recognition system. [3] The performance of PCA depends on the task statement, the subspace distance metric. The performance of ICA depends on the task, so the algorithm is used to approximate ICA. The goal of this paper is to compare a canonical subspace projection technique Principal Component 
Analysis (PCA) to a newer technique, Independent Component Analysis ICA. [4, 5, and 6] Especially in Face Recognition PCA is popular method and ICA is originally developed for separating mixed audio signals into independent sources. In the Figure 1, the learning stages for Face Recognition System are shown. Initially, it collects face images as an input from the database. After that, feature extraction is happened by the processing of face images. In the next step, learning algorithm is used and finally kept images in training database for the testing stage. These steps are shown below.

Face collection \& input

Pre-processing of face image

Feature Extraction

Learning Algorithm

Saving the image in training database for the testing stage.

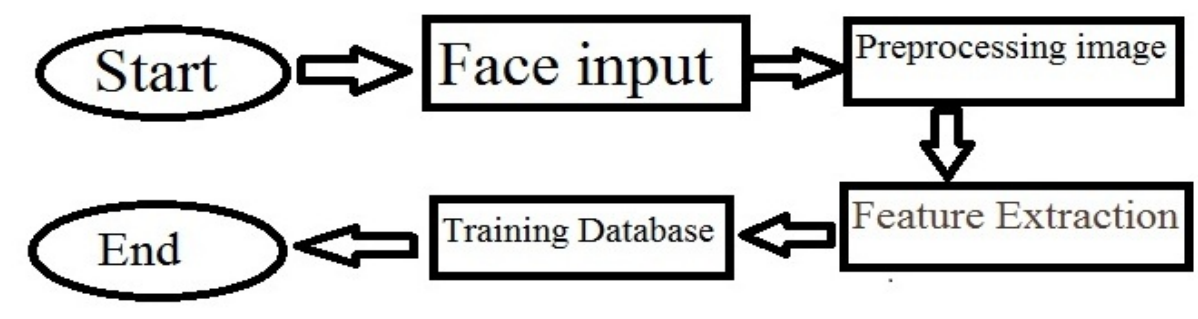

Figure 2. Learning stage for Face Recognition.

In the Figure 2, at first take input of face image for matching. Then processes the face image for feature extraction. Finally applied the recognition algorithm and provide the output result for successfully recognised or unsuccessfully recognized. These steps are shown below.

Face (input)

Pre-processing of the face image

Feature Extraction

Recognition Algorithm

$\square$ Success or Failure (output)

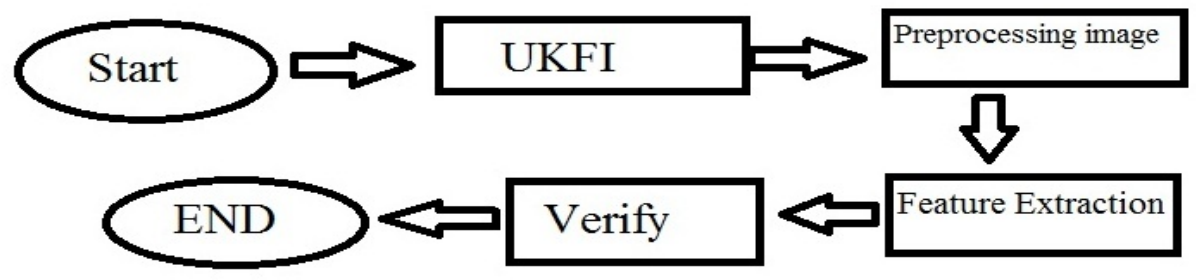

Figure 2. Testing stage for Face Recognition.

But actually the percentage of Face Recognition system of ICA is still not so good. For this reason, Face Recognition system is still now researched. In this paper, the algorithm of PCA and ICA are proposed. PCA is more powerful and sharp than ICA. Due to the percentage reason, the proposed algorithm is increasing noise free system for Face Recognition, which is more standard than previous. 


\section{PCA \& ICA}

For PCA all images must be same size and resolution and one image is treated as one vector. Gallery images represents as matrix and images are set are stored in a single matrix. The average image has to be calculated and then subtracted from each original image. Covariance matrix is calculated by eigenvectors and Eigen values. These eigenvectors are called Eigen face and its remove the unsuccessful information. Besides, it fester the face structure into the uncollected component. A Block Diagram of Face Recognition System using Principle component analyses are shown in Figure 3:

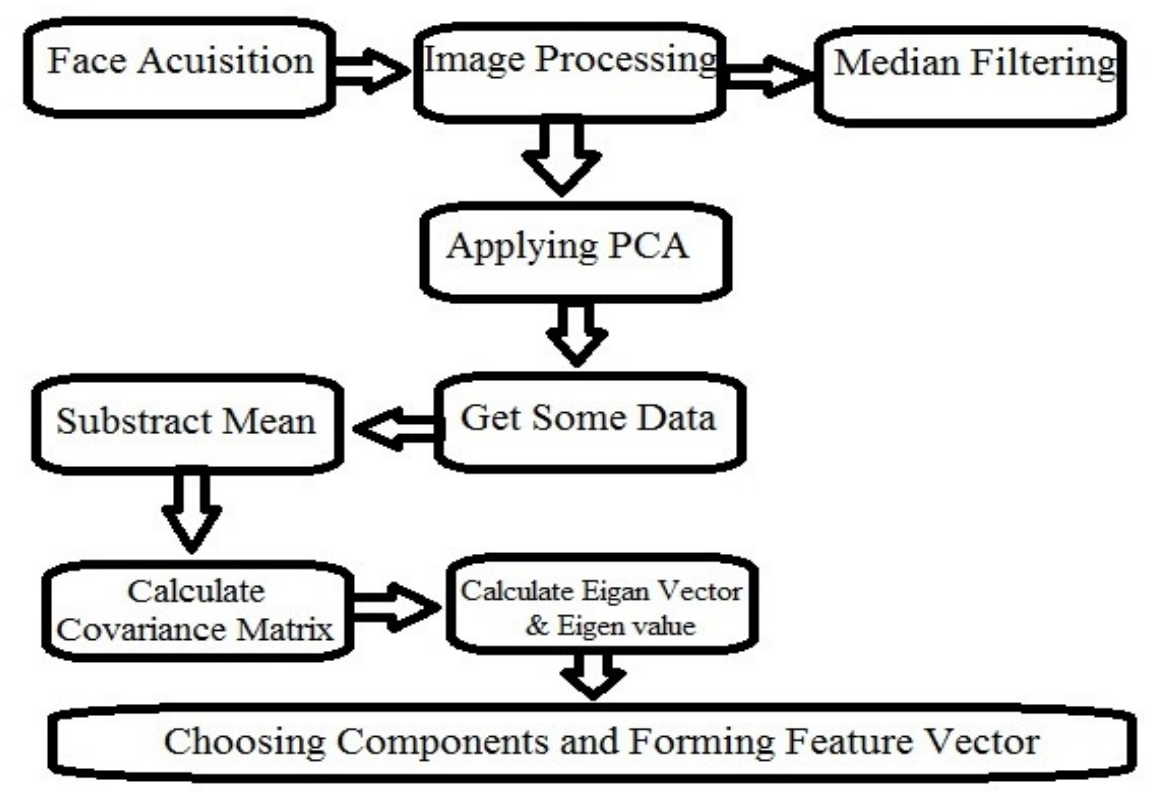

Figure 3. Block diagram of PCA

ICA of a random vector searches for a linear transformation which minimizes the statistical dependence between its components. ICA basis vectors are more spatially localized than their PCA counterparts Evaluations on localized recognition tasks, such as recognizing expressions, may produce significantly different results. [7] Basically ICA is also good for Face Recognition system. Local representation provides robustness to partial occlusions and local distortions because successful Face Recognition can be achieved by representing some important facial parts that correspond to feature regions such as eyes, eye brows, nose and lips. This "recognition by parts" paradigm has been popular in the object recognition research because the approach can be successfully applied to the problem of object recognition with occlusion. [8] The first ICA method computes Independent Components by maximizing non-Gaussianity of whitened data distribution using a kurtosis maximization process. The kurtosis measures the non-Gaussianity and the sparseness of the face representations. [9] Here this system, need to an input image. After the input image it's calculate the Eigen values of training images. Then select a test image or original image. Apply ICA algorithm in the test image. Then get a mean image from the test image. If it matches the training images, the result found otherwise not shown in Figure 4. 
International Journal of Control, Automation, Communication and Systems (IJCACS), Vol.1, No.1, January 2016

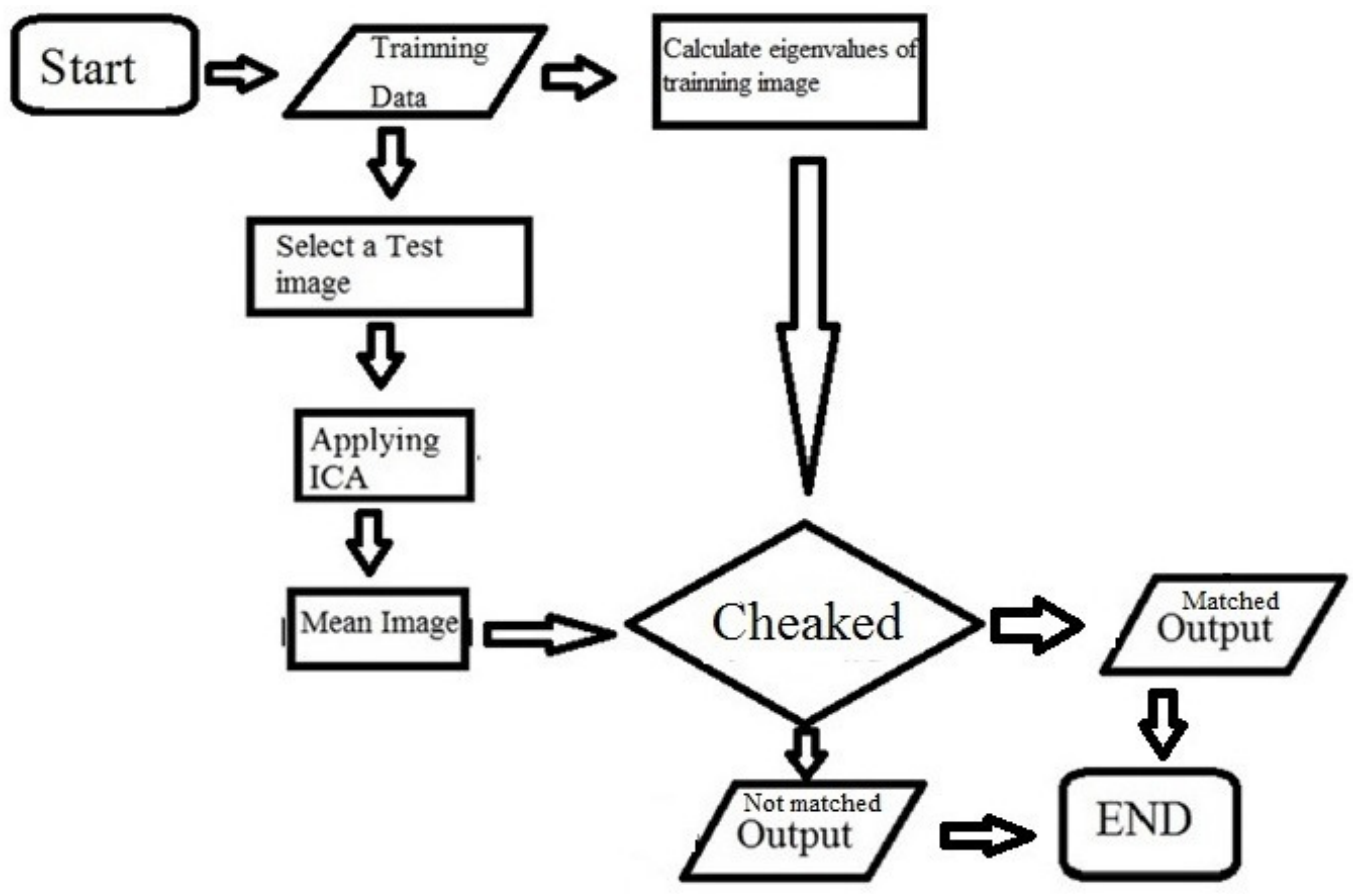

Figure 4. Block diagram of ICA

\section{Proximity of PCA}

For Face Recognition PCA is probably the most widely subspace technique. For PCA,

Obtain $\mathrm{M}$ training images $I_{1}, I_{2}, \ldots \ldots . . I_{M}$ because the images are cantered.

On this formula below, each image $I_{i}$ represented as vector

$$
\left[\begin{array}{lll}
a_{11} & a_{12} & a_{1 N} \\
a_{21} & a_{22} & a_{2 N} \\
a_{N 1} & a_{N 2} & a_{N N}
\end{array}\right] \underset{\mathbf{N}}{\longrightarrow} \underset{\mathbf{N}}{\longrightarrow}\left[\begin{array}{l}
a_{11} \\
a_{1 N} \\
a_{2 N} \\
a_{N N}
\end{array}\right] \mathbf{Z} \mathrm{T}_{\mathrm{i}}
$$

Now find out the average face vector.

$$
\Psi=1 / M \sum_{i=1}^{M} T_{i}
$$




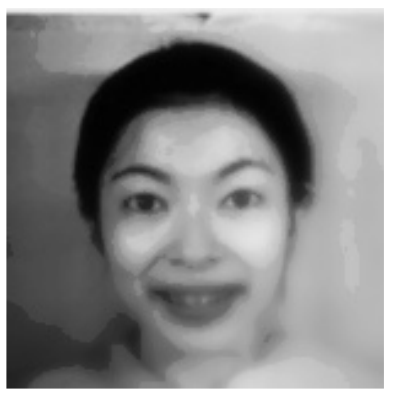

Figure 5. Mean Image

Subtract the mean face from each face vector, to get a set of vectors.

$$
\Phi_{i}=T_{i}-\Psi
$$

Find the covariance matrix $\mathrm{C}=\mathrm{AA}^{\mathrm{T}}$ Where,

$$
A=\left[\Phi_{1}, \Phi_{2}, \ldots . . \Phi_{N}\right]
$$

But $C=N^{2} * N^{2}$ and $A=N^{2} * M$, here $\mathrm{C}$ is huge.

Then find out the, $\mathrm{L}=\mathrm{A}^{\mathrm{T}} \mathrm{A}=\mathrm{M}^{*} \mathrm{M}$

Now can get $\mathrm{V}$ eigenvector and then calculate the eigenvector of $\mathrm{C}$ using this equation (5),

$\mathrm{U}_{i}=\mathrm{AV}_{i}$
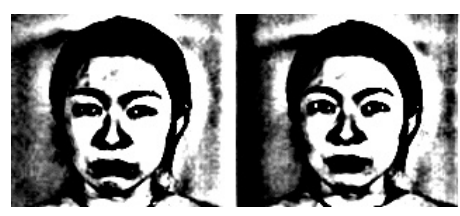

(5)

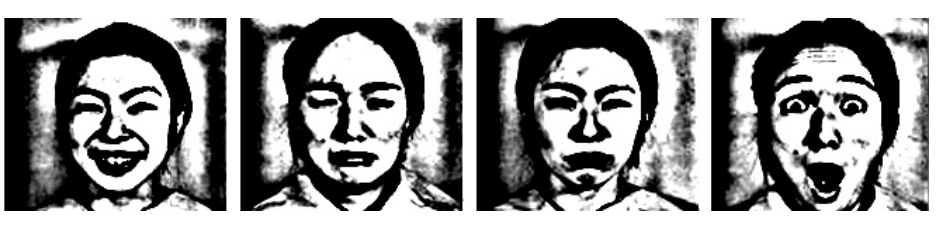

Figure 6. Eigen Faces

Weight calculation for each training images using this formula (6),

$W_{j}=U_{j}^{T} \Phi_{i}$

At last calculate the individual weight vector for each training images using the equation (7),

$\Omega_{i}=\left[\begin{array}{l}w_{1} \\ w_{2} \\ w_{N}\end{array}\right]$

For test image also calculated, $\Omega$. At the last step distance measured from test image to every training image using Euclidean or City blocks distance. The result is found whose distance is minimum.

$$
\text { Eudist }=\sqrt{(\text { test }- \text { train })^{2}}
$$



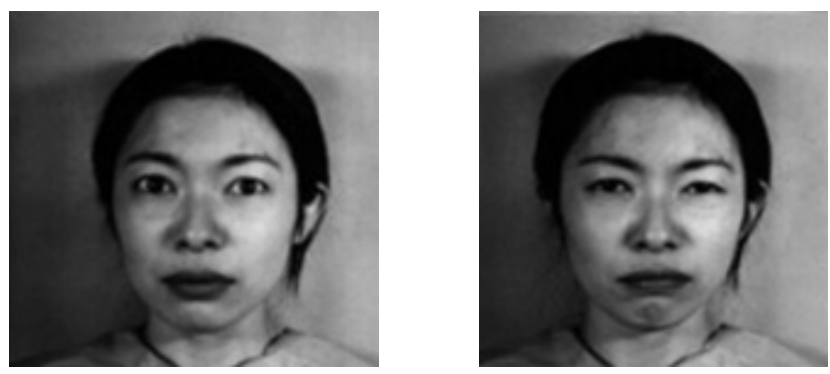

Figure 7. Face Recognized using PCA

\section{Proximity of ICA}

Independent Component Analysis (ICA), a generalization of PCA, is one such method. At first it has number of source $\mathbf{K}$. From the $\mathbf{K}$ source, here found the training image which number is $\mathbf{n}$. Here, $\mathbf{X = A S}$. The De-mixing matrix is $\mathbf{W} . \mathbf{W}$ is an inverse of Recovered the ICA, $\mathbf{U}=\mathbf{W X}$.

$\mathbf{W}$ is comes from the Informix algorithm proposed. $\mathbf{W}$ is selected randomly. $\mathbf{W}$ is increased by this algorithm is given bellow.

$$
f_{i}(U)=1 / 1+e^{-U}
$$

Where,

$$
Y_{i}=f^{\prime \prime}{ }_{i}\left(U_{i}\right) / f^{\prime}{ }_{i}\left(U_{i}\right)
$$

$\Delta W=\left(I+Y^{\prime} U^{T}\right) W$

Here the identity matrix and the logistic transfer function gives,

$$
Y_{i}=\left(1-2 Y_{i}\right)
$$

$\mathbf{X}$ are the training set of images.The whitening matrix is comes from the equation (13),

$W_{z}=2 *(\operatorname{cov}(X))^{(-1 / 2)}$

When the inputs to ICA are the "sphere" data, the full transform matrix $\mathbf{W}_{i}$ is the product of the sphering matrix and the matrix learned by ICA,

$$
W_{I}=W^{*} W_{z}
$$

The PC representation of the set of zero-mean images in $\mathbf{X}$ based on $\boldsymbol{P}_{\boldsymbol{m}}$ is defined as,

$$
R_{m}=X P_{m}
$$


International Journal of Control, Automation, Communication and Systems (IJCACS), Vol.1, No.1, January 2016

For train image,

$$
B=R_{m} W_{I}^{-1}
$$

For test image,

$$
R_{\text {test }}=X_{\text {test }} P_{m} \quad B_{\text {test }}=R_{\text {test }} W_{I}^{-1}
$$

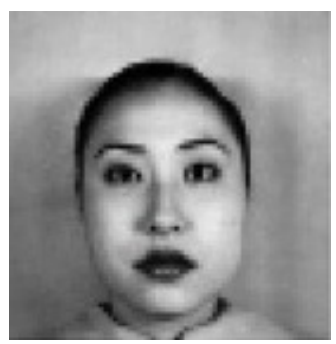

Figure 8. Test image for ICA

Calculate the cosine matrix between test and training set by using this equation (17),

$$
c=B_{\text {test }} * B_{\text {train }} /\left\|B_{\text {test }}\right\|\left\|B_{\text {train }}\right\|
$$

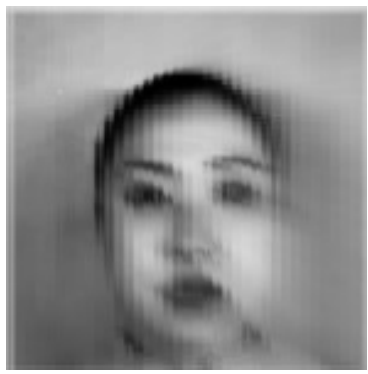

Figure 9. Cosine matrix of an image

Euclidean Distance measure between training and testing image using the cosine angle $\boldsymbol{c}=\boldsymbol{\alpha}$. same as PCA.

$$
d=\sqrt{(\text { test }- \text { train })^{2}-2 \cos (c)}
$$
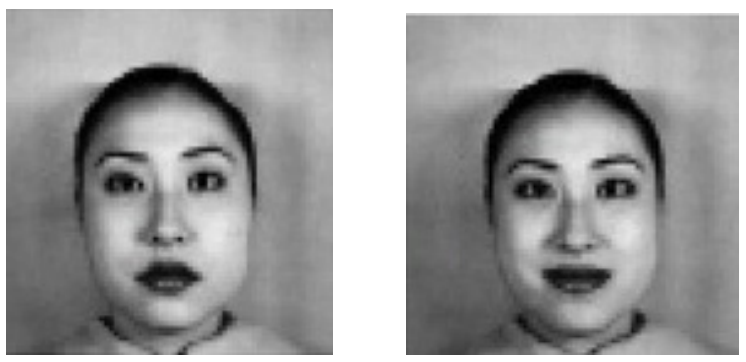

Figure 10. Face Recognised by ICA 
International Journal of Control, Automation, Communication and Systems (IJCACS), Vol.1, No.1, January 2016

\section{PERFORMANCE \& RESUlt ANALYSiS}

For the data Analysis JEFFE database are used. For the analysis, machine has got random results for random number of images. For ICA and PCA, the results are shown below table 1 and table 2 .

Table 1: Competency Calculation for ICA

\begin{tabular}{|l|l|l|l|l|}
\hline $\begin{array}{l}\text { No. of Face } \\
\text { Image }\end{array}$ & 15 & 25 & 35 & 45 \\
\hline $\begin{array}{l}\text { Successfully Recognized } \\
\text { Face Image }\end{array}$ & 14 & 23 & 31 & 40 \\
\hline Unrecognized Face Image & 1 & 2 & 4 & 5 \\
\hline COMPETENCY (\%) & $94 \%$ & $92 \%$ & $88 \%$ & $89 \%$ \\
\hline
\end{tabular}

Table 1 showed that $90.75 \%$ Competency of Face Recognition System by using ICA (Independent Component Analysis).

Table 2: Competency Calculation for PCA

\begin{tabular}{|l|l|l|l|l|}
\hline $\begin{array}{l}\text { No. of Face } \\
\text { Image }\end{array}$ & 15 & 25 & 35 & 45 \\
\hline $\begin{array}{l}\text { Successfully Recognized } \\
\text { Face Image }\end{array}$ & 15 & 23 & 33 & 43 \\
\hline Unrecognized Face Image & 0 & 2 & 2 & 4 \\
\hline COMPETENCY (\%) & $100 \%$ & $92 \%$ & $94 \%$ & $93 \%$ \\
\hline
\end{tabular}

Table 2 showed that $94.75 \%$ Competency of Face Recognition System by using PCA (Principal Component Analysis). If Plot those data into a graph then the graph is shown below Figure 11 and also an efficiency calculation graph are shown Figure 12. 
International Journal of Control, Automation, Communication and Systems (IJCACS), Vol.1, No.1, January 2016

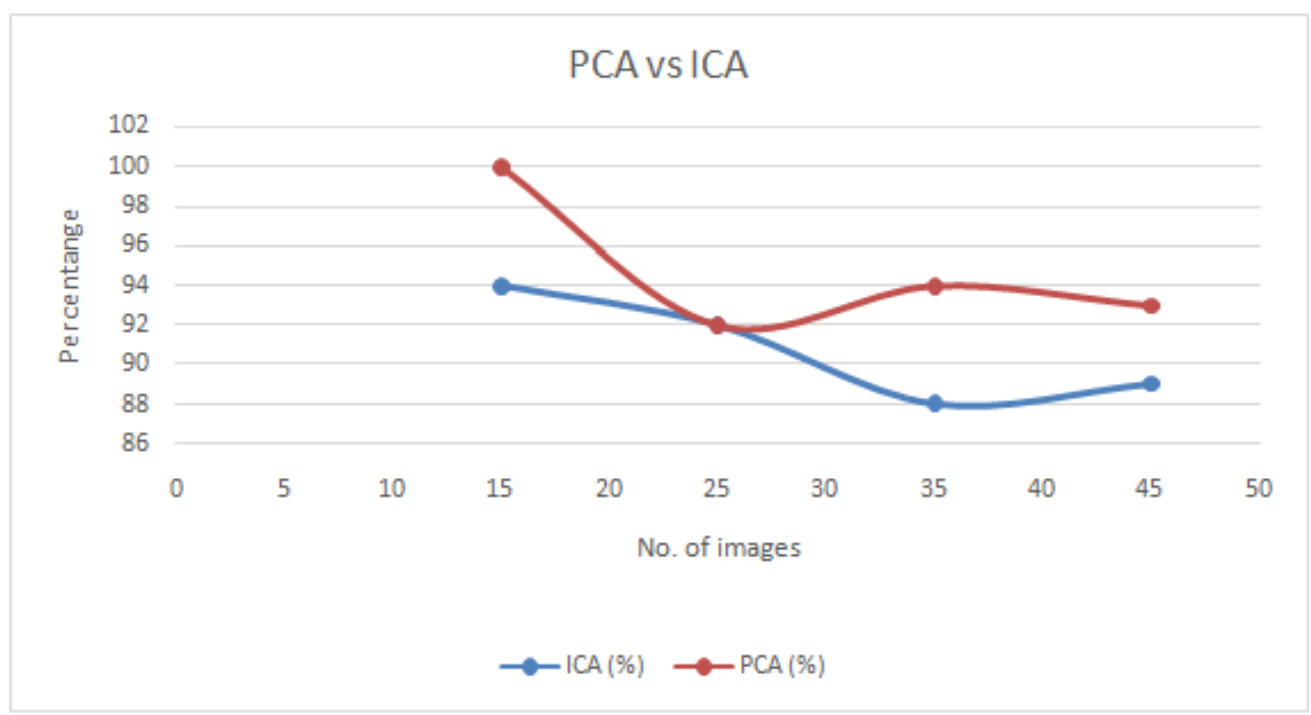

Figure 11. PCAvs ICA Graphs

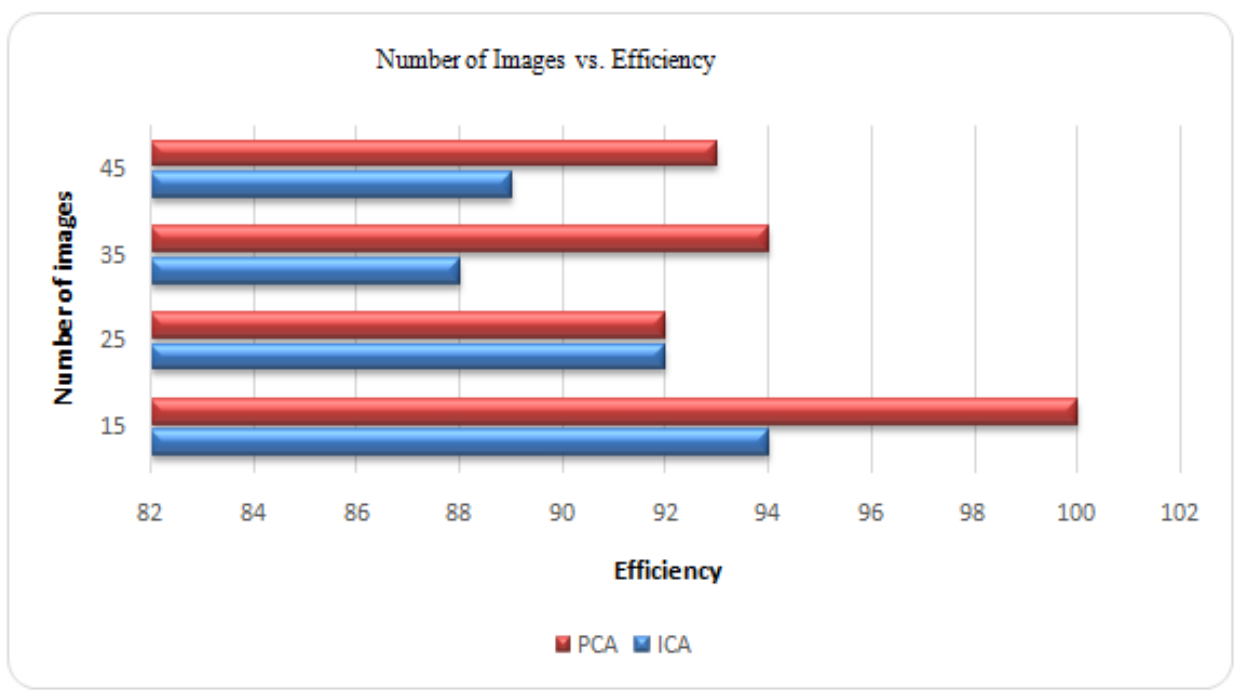

Figure 12.PerformanceChartfor ICA and PCA

\section{Conclusions}

Face Recognition System using the concept of Principle Component Analysis (ICA) and Independent Component Analysis has been discussed. The maximum ability for Face Recognition System by using Principal Component Analysis (PCA) is 94.75\% and the minimum efficiency for Face Recognition System by using Independent Component Analysis is 90.75\%. For this algorithm and database system another important part is camera, cause if good resolution digital camera or good resolution analogue camera is used, the results could be increased and it will give better result. Otherwise it can't carry good result for this system. Principal Component Analysis (PCA) is very safe and better system for search an image from training database. On the other hand Independent Component Analysis (ICA) is good system but critical for comfortable uses. 
International Journal of Control, Automation, Communication and Systems (IJCACS), Vol.1, No.1, January 2016

\section{REFERENCES}

[1] Mahima Agrawal1,Shubangi.D.Giripunje2,P.R.Bajaj3, "Recognizing Facial Expression using PCA and GeneticAlgorithm", International Journal of Computer \& Communication Technology, April 2011.

[2] Bruce A. Draper,a,* KyungimBaek,b Marian Stewart Bartlett,c and J. Ross Beveridge (Computer Vision and Image Understanding 91 (2003) 115-137)

[3] Önsen TOYGAR Adnan ACAN ,\#Face recognition using PCA,LDA and ICA approaches on colored images\|, Journal Of Electrical and Electronics Engineering, vol-13,2003

[4] Turk, M. and A. Pentland, Eigenfaces for Recognition. Journal of Cognitive Neuroscience, 1991. 3(1): p. 71-86

[5] Comon, P., Independent component analysis; A new concept? Signal Processing, 1994. 36(3): p. $287-$ 314.

[6] Bell, A.J. and T.J. Sejnowski, An informationmaximization Approach to Blind Separation and Blind Deconvolution. Neural Computation, 1995. 7(6): p. 1129-1159.

[7] Donato, G., et al., Classifying Facial Actions. IEEE Transactions on Pattern Analysis and Machine Intelligence, 1999. 21(10): p. 974-989

[8] A. P. Pentland, "Recognition by parts," IEEE Proceedings of the First International Conference on Computer Vision, pp. 612-620, 1987.

[9] A. J. Bell and T. J. Sejnowski, "The Independent Components of Natural Scenes are Edge FILTERS," VISION RESEARCH, VOL. 37, NO. 23, PP. 3327-3338, 1997.

\section{Authors}

Md. Shahjahan Kabir is a faculty member of CSE in Dhaka International University. He has got B.Sc. from RUET. He has online publications in the different international journals.

Md. Solaiman Hossain is a student of B.Sc. at CSE in Dhaka International University.

Dr. Rabiul Islam is currently an Associate professor at CSE in RUET. He has already completed his B.Sc., M.Sc. and PhD. from RUET. He has lots of research and publications. He has already published 65 online publications in different international journals. 